\title{
Robust Observer Design for Switched Positive Linear System with Uncertainties
}

\author{
Yanke Zhong and Tefang Chen \\ School of Information Science and Engineering, Central South University, Changsha 410075, China \\ Correspondence should be addressed to Yanke Zhong; zhongyanke1981@163.com
}

Received 10 January 2014; Revised 20 March 2014; Accepted 18 April 2014; Published 7 May 2014

Academic Editor: Jose L. Gracia

Copyright ( 2014 Y. Zhong and T. Chen. This is an open access article distributed under the Creative Commons Attribution License, which permits unrestricted use, distribution, and reproduction in any medium, provided the original work is properly cited.

This paper is concerned with the design of a robust observer for the switched positive linear system with uncertainties. Sufficient conditions of building a robust observer are established by using the multiple copositive Lyapunov-krasovskii function and the average dwell time approach. By introducing an auxiliary slack variable, these sufficient conditions are transformed into LMI (linear matrix inequality). A numerical example is given to illustrate the validities of obtained results.

\section{Introduction}

The switched system is a type of hybrid dynamical system, which is composed of several subsystems and a switching law [1]. The switching law governs the switches between subsystems. The switched positive system is a special kind of switched system, whose state and output are nonnegative whenever the initial state and input are nonnegative. In practice, many systems can be modeled as switched positive systems, such as communication system [2], formation flying [3], and viral mutation [4].

Recently, the switched positive system has attracted a lot of attention. As the stability and stabilization problems are basic problems for control systems, the obtained results mainly focus on them [5-11]. Most of the obtained results are sufficient conditions. However, it should be pointed out that Benzaouia and Tadeo proposed the necessary and sufficient condition for the existence of a stabilization controller [12]. In practice, system state may not be measurable. In this case, the problem of building a state observer for switched system is very significant. Considerable attention has been devoted to this problem. In [13], the observers were designed by using the common Lyapunov function and the multiple Lyapunov function, respectively. In [14], an effective method was used to build an observer for the switched linear system with state jumps. Taking uncertainties into account, Xiang et al. designed a robust observer for the switched nonlinear system [15]. However, since the state of switched positive system is positive, the state observer must also be positive. The straightforward application of the above methods to the switched positive system may result in meaningless results [16]. Thus, the state of observer should be restricted to be positive. Rami et al. designed positive observers for the linear continuous positive system [17] and the linear discrete positive system [18]. In $[19,20]$, a positive observer was built for the positive system with time delays. For the positive linear system with interval uncertainties, Shu et al. proposed necessary and sufficient conditions for the existence of a positive observer. Furthermore, these conditions were described by system matrices. Hence complex matrix decomposition was avoided [21]. Although these results are concerned with the positive system observers, they also contribute to the design of switched positive system observers.

In practice, switched systems are commonly subjected to time delays which have great impacts on the performances of systems. Some published papers have discussed time delay in detail [22-25]. Besides, model uncertainties universally exist in systems and may deteriorate the performances of systems. 
Thus, the state observer should be robust to model uncertainties. In [26, 27], two different methods were proposed to deal with the polytypic uncertainty. In [28], a robust observer was built for the switching discrete system with uncertainties. Furthermore, by introducing slack variables, the obtained results were presented in form of LMI.

This paper focuses on the robust state observer of switched positive system with uncertainties and time-varying delay. The main contributions of this paper are summarized as follows. (1) Taking model uncertainties into account, the robust observer is obtained; (2) the sufficient conditions of building a robust observer are proposed in form of LMI; (3) the designed state observer is positive.

The rest of this paper is organized as follows. Some necessary definitions and lemmas are introduced in Section 2. In Section 3, a robust positive observer is designed for the switched positive linear system. In Section 4, a numerical example is given. The conclusions are presented in Section 5 .

Notations. $R^{n}\left(R_{+}^{n}\right)$ stands for $n$-dimensional real (positive) vector space; $R^{n \times n}$ denotes the space of $n \times n$ matrices with real entries; $M$ represents Metzler matrices whose off diagonal entries are nonnegative; $A \succ 0(A \succeq 0, A \prec 0)$ implies that all elements of matrix $A$ are positive (nonnegative and negative); define $\|x\|=x^{T} x=\left\{x_{1}^{2}+\cdots+x_{n}^{2}\right\}$, where $x_{i}$ is the $i$ th element of vector $x \in R^{n}$; define $\underline{m}=\{1, \ldots, m\}$ and $\underline{n}=\{1, \ldots, n\}$, where $m$ and $n$ are arbitrary positive integers.

\section{Problem Statements and Preliminaries}

Consider the following switched linear system:

$$
\begin{gathered}
\dot{x}(t)=\left(A_{\sigma(t)}+\Delta A_{\sigma(t)}\right) x(t)+\left(B_{\sigma(t)}+\Delta B_{\sigma(t)}\right) x(t-d(t)) \\
+F_{\sigma(t)} u(t), \quad 0 \leq d(t) \leq \tau, \dot{d}(t) \leq d \leq 1, \\
x(t)=\varphi(t), \quad t \in[-\tau, 0], \\
y(t)=C_{\sigma(t)} x(t),
\end{gathered}
$$

where $x(t) \in R^{n}$ is the system state; $d(t)$ denotes time-varying delay; $y(t)$ is the output of system; $u(t) \geq 0$ is the control input; $\sigma(t) \in \underline{m}$ is the switching law which is a piecewise continuous function; $\varphi(t) \geq 0$ is the continuous vector-valued initial function; $\tau$ and $d$ are known positive constants; the model uncertainties $\Delta A_{\sigma(t)}$ and $\Delta B_{\sigma(t)}$ are norm bounded, described by $\left[\begin{array}{ll}\Delta A_{\sigma(t)} & \Delta B_{\sigma(t)}\end{array}\right]=D_{\sigma(t)} G_{\sigma(t)}\left[\begin{array}{ll}E_{\sigma(t) 1} & E_{\sigma(t) 2}\end{array}\right]$; $G_{\sigma(t)}$ is unknown matrix satisfying $G_{\sigma(t)}^{T} G_{\sigma(t)} \leq I ; D_{\sigma(t)}, E_{\sigma(t) 1}$, and $E_{\sigma(t) 2}$ are known matrices; $A_{\sigma(t)} \in M, B_{\sigma(t)} \geq 0, F_{\sigma(t)} \geq 0$, and $C_{\sigma(t)} \geq 0$ are known system matrices with appropriate dimensions; besides, $\left(A_{\sigma(t)}+\Delta A_{\sigma(t)}\right) \in M,\left(B_{\sigma(t)}+\Delta B_{\sigma(t)}\right) \geq 0$.

Next, some necessary definitions and lemmas are introduced.

Definition 1. For any initial state $x_{0} \geq 0$ and $u(t) \geq 0$, if the corresponding trajectories $x(t) \geq 0$ and $y(t) \geq 0$ hold for $t \geq 0$, then the system (1) is called switched positive linear system [5].
Definition 2. If there exist positive constants $\alpha>0$ and $\beta>0$ such that

$$
\|x(t)\| \leq \alpha e^{-\beta\left(t-t_{0}\right)} \sup _{t_{0} \in[-\tau, 0]}\left\|x\left(t_{0}\right)\right\|, \quad \forall t \geq t_{0},
$$

then the system (1) is globally uniformly exponentially stable (GUES) under switching law $\sigma(t)[16]$.

Definition 3. For $T \geq t \geq 0$, let $N_{\sigma(t)}(t, T)$ denote the switching number of $\sigma(t)$ over $(t, T]$. If

$$
N_{\sigma(t)}(t, T) \leq N_{0}+\frac{T-t}{\tau_{a}}
$$

holds for $\tau_{a} \geq 0$ and an integer $N_{0} \geq 0$, then $\tau_{a}$ is called the average dwell time (ADT) [6].

Assumption 4. The state trajectory of system (1) is continuous everywhere. In other words, state variable does not jump at switching instants.

Lemma 5. System (1) is positive if and only if $\left(A_{\sigma(t)}+\Delta A_{\sigma(t)}\right) \in$ $M,\left(B_{\sigma(t)}+\Delta B_{\sigma(t)}\right) \geq 0, u(t) \succeq 0, x_{0} \geq 0, F_{\sigma(t)} \geq 0$, and $C_{\sigma(t)} \geq 0$ hold for $\sigma(t) \in \underline{m}[16]$.

Lemma 6 (see [16]). For matrices $D$ and $E$ and symmetric matrix $Y, Y+D F E+E^{T} F^{T} D^{T}<0$ holds for $F^{T} F \leq I$ if and only if there exists a positive constant $\varepsilon$ such that

$$
Y+\varepsilon D D^{T}+\varepsilon^{-1} E^{T} E<0 .
$$

\section{Robust Observer Design}

In this section, we focus on the design of a robust observer for system (1). According to the structure of system (1), the desired observer is written as

$$
\begin{gathered}
\dot{\hat{x}}(t)=A_{\sigma(t)} \widehat{x}(t)+B_{\sigma(t)} \widehat{x}(t-d(t))+F_{\sigma(t)} u(t) \\
+H_{\sigma(t)}(y(t)-\widehat{y}(t)), \\
\widehat{x}(t)=0, \quad t \in[-\tau, 0], \\
\hat{y}(t)=C_{\sigma(t)} \widehat{x}(t),
\end{gathered}
$$

or, equivalently,

$$
\begin{gathered}
\dot{\hat{x}}(t)=\left(A_{\sigma(t)}-H_{\sigma(t)} C_{\sigma(t)}\right) \widehat{x}(t)+B_{\sigma(t)} \widehat{x}(t-d(t)) \\
+F_{\sigma(t)} u(t)+H_{\sigma(t)} C_{\sigma(t)} x(t), \\
\widehat{x}(t)=0, \quad t \in[-\tau, 0] \\
\hat{y}(t)=C_{\sigma(t)} \widehat{x}(t),
\end{gathered}
$$

where $\widehat{x}(t)$ and $\widehat{y}(t)$ denote the state and output of observer, respectively, and $H_{\sigma(t)}$ is the gain matrix to be determined.

According to Lemma 5 and the given conditions, system (1) is a switched positive linear system. Since the state of system (1) is positive, the desired observer is required to be positive. Thus, $H_{\sigma(t)}$ should satisfies the following conditions:

$$
\begin{gathered}
\left(A_{\sigma(t)}-H_{\sigma(t)} C_{\sigma(t)}\right) \in M \quad \text { for } \sigma(t) \in \underline{m}, \\
H_{\sigma(t)} C_{\sigma(t)} \geq 0 \quad \text { for } \sigma(t) \in \underline{m} .
\end{gathered}
$$


Define $\widetilde{x}(t)=x(t)-\widehat{x}(t)$ and $\widetilde{y}(t)=y(t)-\hat{y}(t)$. From (1) and (6), error system (8) is obtained as follows:

$$
\begin{gathered}
\dot{\tilde{x}}(t)=\left(A_{\sigma(t)}-H_{\sigma(t)} C_{\sigma(t)}\right) \tilde{x}(t)+B_{\sigma(t)} \tilde{x}(t-d(t)) \\
+\Delta A_{\sigma(t)} x(t)+\Delta B_{\sigma(t)} x(t-d(t)) \\
\tilde{x}(t)=\varphi(t), \quad t \in[-\tau, 0] \\
\tilde{y}(t)=C_{\sigma(t)} \tilde{x}(t) .
\end{gathered}
$$

Define

$$
\begin{gathered}
\bar{x}(t)=\left[\begin{array}{l}
x(t) \\
\tilde{x}(t)
\end{array}\right], \quad \bar{u}(t)=\left[\begin{array}{c}
u(t) \\
0
\end{array}\right], \\
\Delta \bar{A}_{\sigma(t)}=\left[\begin{array}{cc}
\Delta A_{\sigma(t)} & 0 \\
\Delta A_{\sigma(t)} & 0
\end{array}\right], \quad \Delta \bar{B}_{\sigma(t)}=\left[\begin{array}{cc}
\Delta B_{\sigma(t)} & 0 \\
\Delta B_{\sigma(t)} & 0
\end{array}\right], \\
\bar{A}_{\sigma(t)}=\left[\begin{array}{cc}
A_{\sigma(t)} & 0 \\
0 & A_{\sigma(t)}-H_{\sigma(t)} C_{\sigma(t)}
\end{array}\right], \\
\bar{B}_{\sigma(t)}=\left[\begin{array}{cc}
B_{\sigma(t)} & 0 \\
0 & B_{\sigma(t)}
\end{array}\right], \quad \bar{F}_{\sigma(t)}=\left[\begin{array}{cc}
F_{\sigma(t)} & 0 \\
0 & 0
\end{array}\right] .
\end{gathered}
$$

From (1) and (8), the augmented system (10) is obtained as follows:

$$
\begin{aligned}
\dot{\bar{x}}(t)= & \left(\bar{A}_{\sigma(t)}+\Delta \bar{A}_{\sigma(t)}\right) \bar{x}(t)+\left(\bar{B}_{\sigma(t)}+\Delta \bar{B}_{\sigma(t)}\right) \bar{x}(t-d(t)) \\
& +\bar{F}_{\sigma(t)} \bar{u}(t) .
\end{aligned}
$$

Let $\bar{D}_{\sigma(t)}^{T}=\left[\begin{array}{ll}D_{\sigma(t)}^{T} & D_{\sigma(t)}^{T}\end{array}\right], \bar{G}_{\sigma(t)}=G_{\sigma(t)}, \bar{E}_{\sigma(t) 1}=$ $\left[\begin{array}{ll}E_{\sigma(t) 1} & 0\end{array}\right]$, and $\bar{E}_{\sigma(t) 2}=\left[\begin{array}{ll}E_{\sigma(t) 2} & 0\end{array}\right]$.

Consequently,

$$
\left[\begin{array}{ll}
\Delta \bar{A}_{\sigma(t)} & \Delta \bar{B}_{\sigma(t)}
\end{array}\right]=\bar{D}_{\sigma(t)} \bar{G}_{\sigma(t)}\left[\begin{array}{ll}
\bar{E}_{\sigma(t) 1} & \bar{E}_{\sigma(t) 2}
\end{array}\right] .
$$

Remark 7. According to Lemma 5, if conditions (7a) and (7b) hold, then system (6) is positive. Furthermore, if system (10) is stable, then $\widetilde{x}(t)$ is converged to zero. This fact implies that the state of system (6) is also converged to state of system (1). Then, system (6) is a positive observer of system (1). Therefore we should choose appropriate $H_{\sigma(t)}$ such that (a) the conditions (7a) and (7b) are satisfied and (b) the system (10) is stable.

Next, we propose two lemmas which are utilized to build an observer for system (1).

Lemma 8. For given constants $\lambda>0$ and $\tau \geq 0$, if there exist symmetric positive definition matrices $Q_{p}, P_{p}$, and $R_{p}$, matrix $H_{p}$, and positive scalar $\varepsilon$ such that

$$
\left[\begin{array}{ccccc}
\Phi_{11} & \Phi_{12} & Q_{p} \bar{F}_{p} & \bar{E}_{p 1}^{T} & Q_{p} \bar{D}_{p} \\
* & \Phi_{22} & 0 & \bar{E}_{p 2}^{T} & 0 \\
* & * & 0 & 0 & 0 \\
* & * & * & -\varepsilon I & 0 \\
* & * & * & * & -\varepsilon^{-1} I
\end{array}\right]<0,
$$

then

$$
\left[\begin{array}{ccc}
S_{11} & Q_{p}\left(\bar{B}_{p}+\Delta \bar{B}_{p}\right)+\frac{e^{-\lambda \tau}}{\tau} R_{p} & Q_{p} \bar{F}_{p} \\
* & -(1-d) e^{-\lambda d} P_{p}-\frac{e^{-\lambda \tau}}{\tau} R_{p} & 0 \\
* & * & 0
\end{array}\right]<0,
$$

where

$$
\begin{gathered}
p \in \underline{m}, \\
\Phi_{11}=\bar{A}_{p}^{T} Q_{p}+Q_{p} \bar{A}_{p}+\lambda Q_{p}+P_{p}+\tau R_{p}-\frac{e^{-\lambda \tau}}{\tau} R_{p}, \\
\Phi_{12}=Q_{p} \bar{B}_{p}+\frac{e^{-\lambda \tau}}{\tau} R_{p}, \\
\Phi_{22}=-(1-d) e^{-\lambda d} P_{p}-\frac{e^{-\lambda \tau}}{\tau} R_{p}, \\
S_{11}=\left(\bar{A}_{p}+\Delta \bar{A}_{p}\right)^{T} Q_{p}+Q_{p}\left(\bar{A}_{p}+\Delta \bar{A}_{p}\right)+\lambda Q_{p}+P_{p} \\
+\tau R_{p}-\frac{e^{-\lambda \tau}}{\tau} R_{p} .
\end{gathered}
$$

Proof. Introduce a new matrix $Y$ which is written as

$$
Y=\left[\begin{array}{ccc}
\Phi_{11} & \Phi_{12} & Q_{p} \bar{F}_{p} \\
* & \Phi_{22} & 0 \\
* & * & 0
\end{array}\right]
$$

According to Schur complement, (12) is equivalent to

$$
\begin{aligned}
& Y+\varepsilon\left[\begin{array}{c}
Q_{p} \bar{D}_{p} \\
0 \\
0
\end{array}\right]\left[\begin{array}{c}
Q_{p} \bar{D}_{p} \\
0 \\
0
\end{array}\right]^{T} \\
& +\varepsilon^{-1}\left[\begin{array}{lll}
\bar{E}_{p 1} & \bar{E}_{p 2} & 0
\end{array}\right]^{T}\left[\begin{array}{lll}
\bar{E}_{p 1} & \bar{E}_{p 2} & 0
\end{array}\right]<0 .
\end{aligned}
$$

By Lemma 6, (16) is equivalent to

$$
\begin{aligned}
Y & +\left[\begin{array}{c}
Q_{p} \bar{D}_{p} \\
0 \\
0
\end{array}\right] \bar{G}_{p}\left[\bar{E}_{p 1} \bar{E}_{p 2} 0\right] \\
& +\left\{\left[\begin{array}{c}
Q_{p} \bar{D}_{p} \\
0 \\
0
\end{array}\right] \bar{G}_{p}\left[\begin{array}{lll}
\bar{E}_{p 1} & \bar{E}_{p 2} & 0
\end{array}\right]\right\}^{T}<0 .
\end{aligned}
$$


Consequently,

$$
\begin{aligned}
& Y+\left[\begin{array}{c}
Q_{p} \bar{D}_{p} \\
0 \\
0
\end{array}\right] \bar{G}_{p}\left[\begin{array}{lll}
\bar{E}_{p 1} & \bar{E}_{p 2} & 0
\end{array}\right] \\
& +\left\{\left[\begin{array}{c}
Q_{p} \bar{D}_{p} \\
0 \\
0
\end{array}\right] \bar{G}_{p}\left[\begin{array}{lll}
\bar{E}_{p 1} & \bar{E}_{p 2} & 0
\end{array}\right]\right\}^{T} \\
& =\left[\begin{array}{ccc}
S_{11} & Q_{p}\left(\bar{B}_{p}+\Delta \bar{B}_{p}\right)+\frac{e^{-\lambda \tau}}{\tau} R_{p} & Q_{p} \bar{F}_{p} \\
* & -(1-d) e^{-\lambda d} P_{p}-\frac{e^{-\lambda \tau}}{\tau} R_{p} & 0 \\
* & * & 0
\end{array}\right]<0 .
\end{aligned}
$$

Therefore (13) holds. This completes the proof.

Note the following problems in Lemma 8. (a) Inequality (12) includes $Q_{p} \bar{A}_{p}$ which involves product terms between $H_{p}$ and $Q_{p}$; (b) $\varepsilon^{-1}$ also exists in inequality (12). Thus, inequality (12) is not a LMI. We propose Lemma 9 to deal with these problems.

Lemma 9. For given constants $\lambda>0$ and $\tau \geq 0$, inequality (12) holds for $p \in \underline{m}$ if there exist symmetric positive definition matrices $Q_{p}, P_{p}$, and $R_{p}$, matrix $T_{p}$, and positive scalars $a$ and $b$ such that

$$
\left[\begin{array}{ccccc}
\Psi_{11} & \Psi_{12} & Q_{p} \bar{F}_{p} & \bar{E}_{p 1}^{T} b & Q_{p} \bar{D}_{p} \\
* & \Psi_{22} & 0 & \bar{E}_{p 2}^{T} b & 0 \\
* & * & 0 & 0 & 0 \\
* & * & * & a-2 b & 0 \\
* & * & * & * & -a
\end{array}\right]<0
$$

where

$$
\begin{aligned}
& \Psi_{11}=T_{p}^{T}+T_{p}+\lambda Q_{p}+P_{p}+\tau R_{p}-\frac{e^{-\lambda \tau}}{\tau} R_{p}, \\
& \Psi_{12}=Q_{p} \bar{B}_{p}+\frac{e^{-\lambda \tau}}{\tau} R_{p}, \\
& \Psi_{22}=-(1-d) e^{-\lambda d} P_{p}-\frac{e^{-\lambda \tau}}{\tau} R_{p} .
\end{aligned}
$$

Proof. Since $a>0$,

$$
(a-b)^{2} a^{-1} \geq 0
$$

It follows that

$$
a-2 b \geq-b a^{-1} b .
$$

Applying (22) to (19), we have

$$
\left[\begin{array}{ccccc}
\Psi_{11} & \Psi_{12} & Q_{p} \bar{F}_{p} & \bar{E}_{p 1}^{T} b & Q_{p} \bar{D}_{p} \\
* & \Psi_{22} & 0 & \bar{E}_{p 2}^{T} b & 0 \\
* & * & 0 & 0 & 0 \\
* & * & * & -b a^{-1} b & 0 \\
* & * & * & * & -a
\end{array}\right]<0 .
$$

Premultiplying diag $\left\{I, I, I, b^{-1}, I\right\}$ and postmultiplying $\operatorname{diag}\left\{I, I, I, b^{-1}, I\right\}$ to $(23)$ yield

$$
\left[\begin{array}{ccccc}
\Psi_{11} & \Psi_{12} & Q_{p} \bar{F}_{p} & \bar{E}_{p 1}^{T} & Q_{p} \bar{D}_{p} \\
* & \Psi_{22} & 0 & \bar{E}_{p 2}^{T} & 0 \\
* & * & 0 & 0 & 0 \\
* & * & * & -a^{-1} & 0 \\
* & * & * & * & -a
\end{array}\right]<0 .
$$

Let $a=\varepsilon^{-1}$ and let $T_{p}=Q_{p} \bar{A}_{p}$. Then, (24) is equivalent to (12). This completes the proof.

Remark 10. In the proof of Lemma 9, the matrix variable $T_{p}$ is employed to replace the term of $Q_{p} \bar{A}_{p}$ which involves $Q_{p} H_{p}$. The slack scalar $\mathrm{b}$ is used to decouple the product terms brought in by $\varepsilon^{-1}$. By this way, inequality (12) is transformed into a standard LMI.

Now, Theorem 11 is proposed for building a robust positive observer.

Theorem 11. For given constants $\lambda>0, \tau \geq 0$, and $\rho \geq 1$, if there exist symmetric positive definition matrices $Q_{p}, P_{p}$, and $R_{p}$, matrix $T_{p}$, and positive scalars $a$ and $b$ such that

$$
\begin{gathered}
{\left[\begin{array}{ccccc}
\Psi_{11} & \Psi_{12} & Q_{p} \bar{F}_{p} & \bar{E}_{p 1}^{T} b & Q_{p} \bar{D}_{p} \\
* & \Psi_{22} & 0 & \bar{E}_{p 2}^{T} b & 0 \\
* & * & 0 & 0 & 0 \\
* & * & * & a-2 b & 0 \\
* & * & * & * & -a
\end{array}\right]<0,} \\
\left(A_{p}\right)_{i, j}-\left(H_{p} C_{p}\right)_{i, j}>0, \quad\left(H_{p} C_{p}\right)_{k, l}>0 \quad \text { for } p \in \underline{m} ; \\
Q_{i} \leq \rho Q_{j}, \quad P_{i} \leq \rho P_{j}, \quad R_{i} \leq \rho R_{j} \quad \text { for } i, j \in \underline{m}, \quad \text { (25) }
\end{gathered}
$$

and the ADT satisfies

$$
\tau_{a}>\frac{\ln \rho}{\lambda}
$$

then system (10) is GUES with an ADT (26) and system (6) is the desired robust positive observer, where $\left(A_{p}\right)_{i, j}$ and $\left(H_{p} C_{p}\right)_{i, j}$ represent the elements in ith row and $j$ th column of $A_{p}$ and $\left(H_{p} C_{p}\right)$, respectively.

Proof. The proof of Theorem 11 is divided into two parts. We prove that (I) system (10) is stable and (II) system (6) is a positive observer of system (1).

(I) System (10) is stable.

Construct multiple copositive Lyapunov-Krasovskii function for system (10) as follows:

$$
V_{\sigma(t)}(t)=V_{\sigma(t), 1}(t)+V_{\sigma(t), 2}(t)+V_{\sigma(t), 3}(t),
$$


where

$$
\begin{aligned}
V_{\sigma(t), 1}(t) & =\bar{x}^{T}(t) Q_{\sigma(t)} \bar{x}(t) \\
V_{\sigma(t), 2}(t) & =\int_{t-d(t)}^{t} e^{\lambda(-t+s)} \bar{x}^{T}(t) P_{\sigma(t)} \bar{x}(t) d s \\
V_{\sigma(t), 3}(t) & =\int_{-\tau}^{0} \int_{t+\theta}^{t} e^{\lambda(-t+s)} \bar{x}^{T}(t) R_{\sigma(t)} \bar{x}(t) d s d \theta
\end{aligned}
$$

Assume that the $p$ th subsystem is activated over $t \in$ $\left[t_{k}, t_{k+1}\right)$. Let $t_{k}$ represent the instant of the $K$ th switching and let $t_{k}^{-}$denote the instant just before $t_{k}$.

Take the derivatives of $V_{p, 1}(t), V_{p, 2}(t)$, and $V_{p, 3}(t)$ with respect to $t$ along the trajectory of system (10) on $t \in\left[t_{k}, t_{k+1}\right)$.

$$
\begin{aligned}
\dot{V}_{p, 1}(t)= & \bar{x}^{T}(t)\left[\left(\bar{A}_{p}+\Delta \bar{A}_{p}\right)^{T} Q_{p}+Q_{p}\left(\bar{A}_{p}+\Delta \bar{A}_{p}\right)\right] \bar{x}(t) \\
& +\bar{x}^{T}(t) Q_{p}\left(\bar{B}_{p}+\Delta \bar{B}_{p}\right) \bar{x}(t-d(t)) \\
& +\bar{x}^{T}(t) Q_{p} \bar{F}_{p} \bar{u}(t) \\
& +\bar{x}^{T}(t-d(t))\left(\bar{B}_{p}+\Delta \bar{B}_{p}\right)^{T} Q_{p} \bar{x}(t) \\
& +\bar{u}^{T}(t) \bar{F}_{p}^{T} Q_{p} \bar{x}(t)+\lambda \bar{x}^{T}(t) Q_{p} \bar{x}(t)-\lambda V_{p, 1}(t)
\end{aligned}
$$

$$
\begin{aligned}
\dot{V}_{p, 2}(t)= & -\lambda V_{p, 2}(t)+\bar{x}^{T}(t) P_{p} \bar{x}(t) \\
& -(1-\dot{d}(t)) e^{-\lambda d(t)} \bar{x}^{T}(t-d(t)) P_{p} \bar{x}(t-d(t)) \\
\leq & -\lambda V_{p, 2}(t)+\bar{x}^{T}(t) P_{p} \bar{x}(t) \\
& -(1-d) e^{-\lambda d \bar{x}^{T}(t-d(t)) P_{p} \bar{x}(t-d(t)),}
\end{aligned}
$$

$$
\begin{aligned}
\dot{V}_{p, 3}(t)= & -\lambda V_{p, 3}(t)+\tau \bar{x}^{T}(t) R_{p} \bar{x}(t) \\
& -\int_{t-\tau}^{t} e^{\lambda(-t+s)} \bar{x}^{T}(s) R_{p} \bar{x}(s) d s \\
\leq & -\lambda V_{p, 3}(t)+\tau \bar{x}^{T}(t) R_{p} \bar{x}(t) \\
& -e^{-\lambda \tau} \int_{t-d(t)}^{t} \bar{x}^{T}(s) R_{p} \bar{x}(s) d s .
\end{aligned}
$$

According to Jensen inequality and (31),

$$
\begin{aligned}
\dot{V}_{p, 3}(t) \leq & -\lambda V_{p, 3}(t)+\tau \bar{x}^{T}(t) R_{p} \bar{x}(t) \\
& -\frac{e^{-\lambda \tau}}{\tau}[\bar{x}(t)-\bar{x}(t-d(t))]^{T} \\
& \times R_{p}[\bar{x}(t)-\bar{x}(t-d(t))] .
\end{aligned}
$$

Noting (29), (30), and (32), hence,

$$
\begin{aligned}
\dot{V}_{p}(t) \leq & -\lambda V_{p}(t)+\left[\begin{array}{c}
\bar{x}(t) \\
\bar{x}(t-d(t)) \\
\bar{u}(t)
\end{array}\right]^{T} \\
& \times\left[\begin{array}{ccc}
S_{11} & Q_{p}\left(\bar{B}_{p}+\Delta \bar{B}_{p}\right)+\frac{e^{-\lambda \tau}}{\tau} R_{p} & Q_{p} \bar{F}_{p} \\
* & -(1-d) e^{-\lambda d} P_{p}-\frac{e^{-\lambda \tau}}{\tau} R_{p} & 0 \\
* & * & 0
\end{array}\right] \\
& \times\left[\begin{array}{c}
\bar{x}(t) \\
\bar{x}(t-d(t)) \\
\bar{u}(t)
\end{array}\right] .
\end{aligned}
$$

We derive from Lemma 8, Lemma 9, (33), (25a), and (25c) that

$$
\begin{aligned}
\dot{V}_{p}(t)+ & \lambda V_{p}(t) \leq 0, \\
V_{p}(t) & \leq e^{-\lambda\left(t-t_{k}\right)} V_{p}\left(t_{k}\right) \\
& \leq \rho e^{-\lambda\left(t-t_{k}\right)} V_{\sigma\left(t_{k-1}\right)}\left(t_{k}^{-}\right) \\
& =\rho e^{-\lambda\left(t-t_{k-1}\right)} V_{\sigma\left(t_{k-1}\right)}\left(t_{k-1}\right) .
\end{aligned}
$$

By iterative calculation, we have

$$
V_{p}(t) \leq \rho^{k} e^{-\lambda\left(t-t_{0}\right)} V_{\sigma\left(t_{0}\right)}\left(t_{0}\right) .
$$

Define $K_{1}=\max _{p \in m} \lambda\left(Q_{p}\right), K_{2}=\max _{p \in m} \lambda\left(P_{p}\right), K_{3}=$ $\max _{p \in \underline{m}} \lambda\left(R_{p}\right)$, and $K_{4}=\min _{p \in \underline{m}} \lambda\left(Q_{p}\right)$, where $\lambda\left(Q_{p}\right)$ represents all eigenvalues of $Q_{p}$ :

$$
\begin{aligned}
V_{\sigma\left(t_{0}\right)}\left(t_{0}\right)= & \bar{x}^{T}\left(t_{0}\right) Q_{\sigma\left(t_{0}\right)} \bar{x}\left(t_{0}\right) \\
& +\int_{t_{0}-d\left(t_{0}\right)}^{t_{0}} e^{\lambda\left(-t_{0}+s\right)} \bar{x}^{T}\left(t_{0}\right) P_{\sigma\left(t_{0}\right)} \bar{x}\left(t_{0}\right) d s \\
& +\int_{-\tau}^{0} \int_{t_{0}+\theta}^{t_{0}} e^{\lambda\left(-t_{0}+s\right)} \bar{x}^{T}\left(t_{0}\right) R_{\sigma\left(t_{0}\right)} \bar{x}\left(t_{0}\right) d s d \theta \\
\leq & \bar{x}^{T}\left(t_{0}\right) K_{1} \bar{x}\left(t_{0}\right) \\
& +\int_{t_{0}-\tau}^{t_{0}} e^{\lambda\left(-t_{0}+s\right)} \bar{x}^{T}\left(t_{0}\right) K_{2} \bar{x}\left(t_{0}\right) d s \\
& +\int_{-\tau}^{0} \int_{t_{0}-\tau}^{t_{0}} e^{\lambda\left(-t_{0}+s\right)} \bar{x}^{T}\left(t_{0}\right) K_{3} \bar{x}\left(t_{0}\right) d s d \theta .
\end{aligned}
$$

Note that $s \in\left[t_{0}-\tau, t_{0}\right]$ and $e^{\lambda\left(-t_{0}+s\right)} \in\left[e^{-\lambda \tau}, 1\right]$; hence,

$$
\begin{aligned}
V_{\sigma\left(t_{0}\right)}\left(t_{0}\right) \leq & \bar{x}^{T}\left(t_{0}\right) K_{1} \bar{x}\left(t_{0}\right)+\int_{t_{0}-\tau}^{t_{0}} \bar{x}^{T}\left(t_{0}\right) K_{2} \bar{x}\left(t_{0}\right) d s \\
& +\int_{-\tau}^{0} \int_{t_{0}-\tau}^{t_{0}} \bar{x}^{T}\left(t_{0}\right) K_{3} \bar{x}\left(t_{0}\right) d s d \theta \\
= & K_{1}\left\|\bar{x}\left(t_{0}\right)\right\|+\tau K_{2}\left\|\bar{x}\left(t_{0}\right)\right\|+\tau^{2} K_{3}\left\|\bar{x}\left(t_{0}\right)\right\| .
\end{aligned}
$$



that

It is derived from (35), (37), and the definition of $V_{\sigma(t)}(t)$

$$
\begin{aligned}
\bar{x}^{T}(t) Q_{\sigma(t)} \bar{x}(t) & \leq V_{\sigma(t)}(t) \\
& \leq \rho^{k} e^{-\lambda\left(t-t_{0}\right)}\left(K_{1}+\tau K_{2}+\tau^{2} K_{3}\right)\left\|\bar{x}\left(t_{0}\right)\right\| .
\end{aligned}
$$

Consequently,

$$
\begin{aligned}
\|\bar{x}(t)\| & \leq \frac{K_{1}+\tau K_{2}+\tau^{2} K_{3}}{K_{4}} \rho^{k} e^{-\lambda\left(t-t_{0}\right)}\left\|\bar{x}\left(t_{0}\right)\right\| \\
& \leq \frac{K_{1}+\tau K_{2}+\tau^{2} K_{3}}{K_{4}} e^{-\left(\lambda-\left(\ln \rho / \tau_{a}\right)\right)\left(t-t_{0}\right)}\left\|\bar{x}\left(t_{0}\right)\right\| .
\end{aligned}
$$

Let $\alpha=\left(K_{1}+\tau K_{2}+\tau^{2} K_{3}\right) / K_{0}$ and $\beta=\lambda-\left(\ln \rho / \tau_{a}\right)$. Obviously, $\alpha>0$. Since $\tau_{a}>(\ln \rho / \lambda), \beta>0$. According to Definition 2, system (10) is GUES with an ADT (26). Hence the estimated state converges to state of system (1). In other words, system (6) is an observer for system (1).

(II) System (6) is a positive observer of system (1).

Since $\left(A_{p}\right)_{i, j}-\left(H_{p} C_{p}\right)_{i, j}>0$ for $p \in \underline{m} ; i, j \in \underline{n} ; i \neq j$, $A_{p}-\left(H_{p} C_{p}\right) \in M$. Furthermore, $\left(H_{p} C_{p}\right)_{k, l}>0$ for $k, l \in$ $\underline{n}$ implies that $\left(H_{p} C_{p}\right)>0$. Hence system (6) is a positive system.

Synthesizing (I) and (II), system (6) is the desired robust positive observer for system (1). This completes the proof of Theorem 11.

Remark 12. $T_{p}$ and $Q_{p}$ can be obtained by solving (25a). Since $T_{p}=Q_{p} \bar{A}_{p}, H_{p}$ can be obtained. If $H_{p}$ satisfies (25b), then $H_{p}$ meets all requirements of design. By this way, the desired robust positive observer is built for system (1).

Remark 13. Note the following problems. (a) Since the conditions (7a) and (7b) are not strictly positive, they are not strict LMI; (b) under the conditions (7a) and (7b), the state and output of system may be always zero; in this case, the obtained result may be useless for engineering practice. Considering these problems, the conditions are replaced by (25b) which is a strict LMI.

\section{Numerical Example}

A numerical example is given to illustrate the validity of the obtained result in this section.

Consider the switched positive linear system with uncertainties and time-varying delay given by

$$
\begin{gathered}
\dot{x}(t)=\left(A_{\sigma(t)}+\Delta A_{\sigma(t)}\right) x(t)+\left(B_{\sigma(t)}+\Delta B_{\sigma(t)}\right) x(t-d(t)) \\
+F_{\sigma(t)} u(t), \quad 0 \leq d(t) \leq \tau, \dot{d}(t) \leq d \leq 1, \\
x(t)=\varphi(t), \quad t \in[-\tau, 0] \\
y(t)=C_{\sigma(t)} x(t),
\end{gathered}
$$

where

$$
\begin{aligned}
& A_{1}=\left[\begin{array}{cc}
-6 & 3 \\
2 & -5
\end{array}\right], \quad B_{1}=\left[\begin{array}{ll}
0.2 & 0.1 \\
0.2 & 0.3
\end{array}\right], \\
& F_{1}=\left[\begin{array}{cc}
0.5 & 0.5 \\
0.2 & 1
\end{array}\right], \quad C_{1}=\left[\begin{array}{ll}
0.05 & 0.01 \\
0.02 & 0.01
\end{array}\right] \text {, } \\
& A_{2}=\left[\begin{array}{cc}
-5 & 2 \\
3 & -4
\end{array}\right], \quad B_{2}=\left[\begin{array}{ll}
0.3 & 0.1 \\
0.2 & 0.2
\end{array}\right], \\
& F_{2}=\left[\begin{array}{ll}
0.1 & 0.2 \\
0.2 & 1.2
\end{array}\right], \quad C_{2}=\left[\begin{array}{ll}
0.01 & 0.02 \\
0.01 & 0.05
\end{array}\right] \text {, } \\
& D_{1}=\left[\begin{array}{cc}
0.3 & 0 \\
0 & 0.2
\end{array}\right], \quad D_{2}=\left[\begin{array}{cc}
0.2 & 0 \\
0 & 0.5
\end{array}\right] \text {, } \\
& E_{1}=\left[\begin{array}{ll}
0.2 & 0.1 \\
0.1 & 0.4
\end{array}\right], \quad E_{2}=\left[\begin{array}{cc}
0.1 & 0.05 \\
0.04 & 0.06
\end{array}\right] \text {, } \\
& \tau=0.4, \quad d=0.8, \quad \varphi(t)=[6,8]^{T}, \quad t \in[-\tau, 0], \\
& u^{T}(t)=[\sin (t)+1 \cos (t)+1] \succeq 0 .
\end{aligned}
$$

Let $\lambda=0.5$ and let $\rho=1.1$. We have $\tau_{a}>0.1906$ from (26). Solving the LMI (25a), we have

$$
\begin{aligned}
T_{1} & =\left[\begin{array}{cccc}
T_{11} & T_{12} & T_{13} & T_{14} \\
* & T_{22} & T_{23} & T_{24} \\
* & * & T_{33} & T_{34} \\
* & * & * & T_{44}
\end{array}\right], \\
Q_{1} & =\left[\begin{array}{cccc}
Q_{11} & Q_{12} & Q_{13} & Q_{14} \\
* & Q_{22} & Q_{23} & Q_{24} \\
* & * & Q_{33} & Q_{34} \\
* & * & * & Q_{44}
\end{array}\right], \\
T_{2} & =\left[\begin{array}{cccc}
T_{11}^{\prime} & T_{12}^{\prime} & T_{13}^{\prime} & T_{14}^{\prime} \\
* & T_{22}^{\prime} & T_{23}^{\prime} & T_{24}^{\prime} \\
* & * & T_{33}^{\prime} & T_{34}^{\prime} \\
* & * & * & T_{44}^{\prime}
\end{array}\right], \\
Q_{2}= & {\left[\begin{array}{cccc}
Q_{11}^{\prime} & Q_{12}^{\prime} & Q_{13}^{\prime} & Q_{14}^{\prime} \\
* & Q_{22}^{\prime} & Q_{23}^{\prime} & Q_{24}^{\prime} \\
* & * & Q_{33}^{\prime} & Q_{34}^{\prime} \\
* & * & * & Q_{44}^{\prime}
\end{array}\right], }
\end{aligned}
$$

where

$$
\begin{array}{rrr}
T_{11}=0.174, & T_{12}=-0.199, & T_{13}=-0.043, \\
T_{14}=0.004, & T_{22}=0.941, & T_{23}=0.084, \\
T_{24}=-0.003, & T_{33}=1.442, & T_{34}=-0.218, \\
T_{44}=-0.15, & T_{11}^{\prime}=-2.043, & T_{12}^{\prime}=1.111,
\end{array}
$$




$$
\begin{array}{ccc}
T_{13}^{\prime}=0.659, & T_{14}^{\prime}=0.4188, & T_{22}^{\prime}=-1.013, \\
T_{23}^{\prime}=-0.789, & T_{24}^{\prime}=-0.151, & T_{33}^{\prime}=0.604, \\
T_{34}^{\prime}=0.448, & T_{44}^{\prime}=0.302, & Q_{11}=-1.9695, \\
Q_{12}=2.8069, & Q_{13}=0.38263, & Q_{14}=-4.6681, \\
Q_{22}=-17.142, & Q_{23}=-0.7126, & Q_{24}=2.4388, \\
Q_{33}=-13.167, & Q_{34}=-6.8238, & Q_{44}=-17.53, \\
Q_{11}^{\prime}=8.531, & Q_{12}^{\prime}=4.953, & Q_{13}^{\prime}=-0.4568, \\
Q_{14}^{\prime}=-0.6424, & Q_{22}^{\prime}=13.88, & Q_{23}^{\prime}=-0.1376, \\
Q_{24}^{\prime}=-0.7494, & Q_{33}^{\prime}=18.189, & Q_{34}^{\prime}=3.3355,
\end{array}
$$

Noting that

$$
T_{p}=Q_{p}\left[\begin{array}{cc}
A_{p} & 0 \\
0 & A_{p}-H_{p} C_{p}
\end{array}\right]
$$

then

$$
H_{1}=\left[\begin{array}{cc}
6.8611 & 8.1389 \\
-8.6849 & 26.712
\end{array}\right], \quad H_{2}=\left[\begin{array}{cc}
30.186 & -8.0745 \\
12.263 & 5.47450
\end{array}\right] .
$$

Consequently,

$$
\begin{gathered}
\left(A_{1}-H_{1} C_{1}\right)=\left[\begin{array}{cc}
-6.3733 & 2.8408 \\
1.4998 & -5.2810
\end{array}\right] \in M, \\
H_{1} C_{1}=\left[\begin{array}{ll}
0.3733 & 0.1592 \\
0.5002 & 0.2801
\end{array}\right] \succeq 0, \\
\left(A_{2}-H_{2} C_{2}\right)=\left[\begin{array}{cc}
-5.2211 & 1.8000 \\
2.8226 & -4.5190
\end{array}\right] \in M, \\
H_{2} C_{2}=\left[\begin{array}{ll}
0.2211 & 0.2000 \\
0.1774 & 0.5190
\end{array}\right] \succeq 0 .
\end{gathered}
$$

Since $H_{1}$ and $H_{2}$ meet all requirements of the design, the design of state observer for system (40) is completed.

The simulation results are shown in Figures 1-3. From them, we can find the following facts.

(1) In Figure 1, it is easy to get that ADT $=1.4286>$ 0.1906 .

(2) In Figure 2, the state of observer approximates the state of original system. This fact is also revealed by Figure 3 in which the state of error system exponentially converges to zero.

(3) The state of observer is positive all the time.

Therefore, the numerical example illustrates the validity of the proposed method.

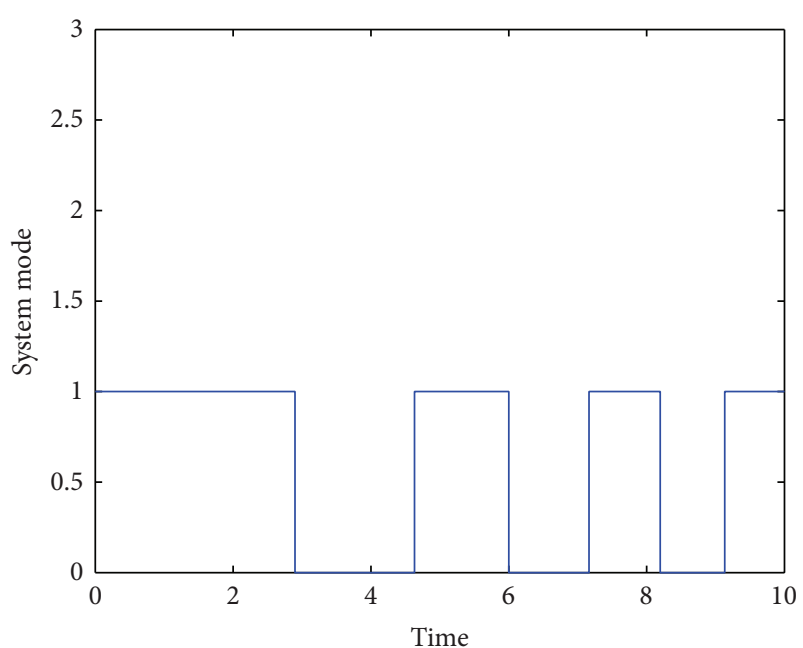

FIGURE 1: The figure of switching law.

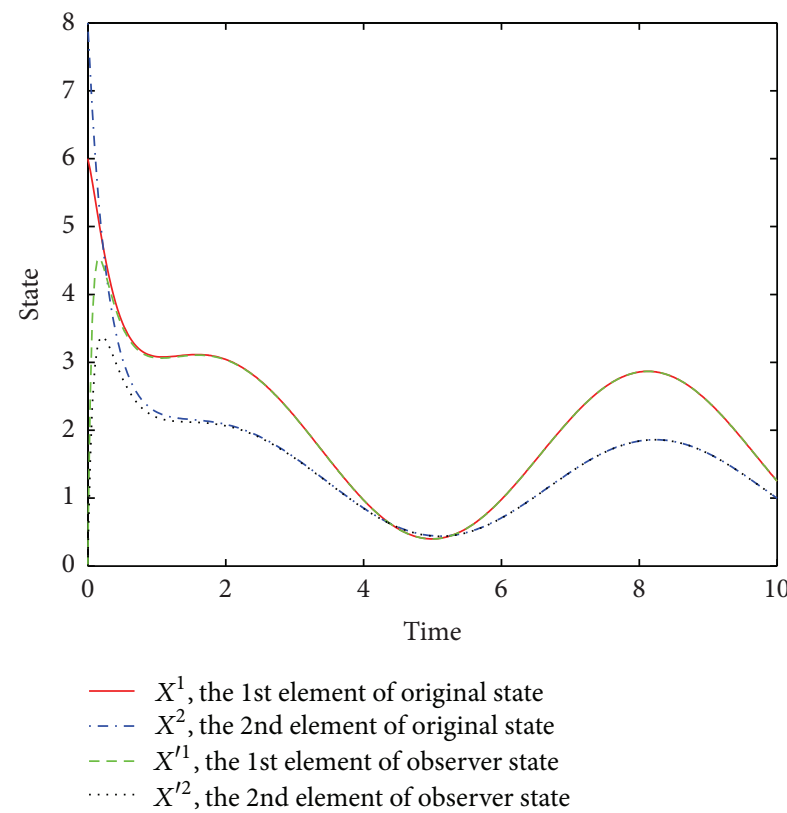

Figure 2: States of observer and original system.

\section{Conclusions}

The design of a robust observer for the switched positive linear system has been investigated in this paper.

(1) In the presence of model uncertainties, the sufficient conditions for the existence of a positive observer are proposed in form of LMI.

(2) The state of observer is positive and converges to the state of original system.

(3) In the future study, the significant task is to investigate fault detection for switched positive linear system with uncertainties based on state observer. 


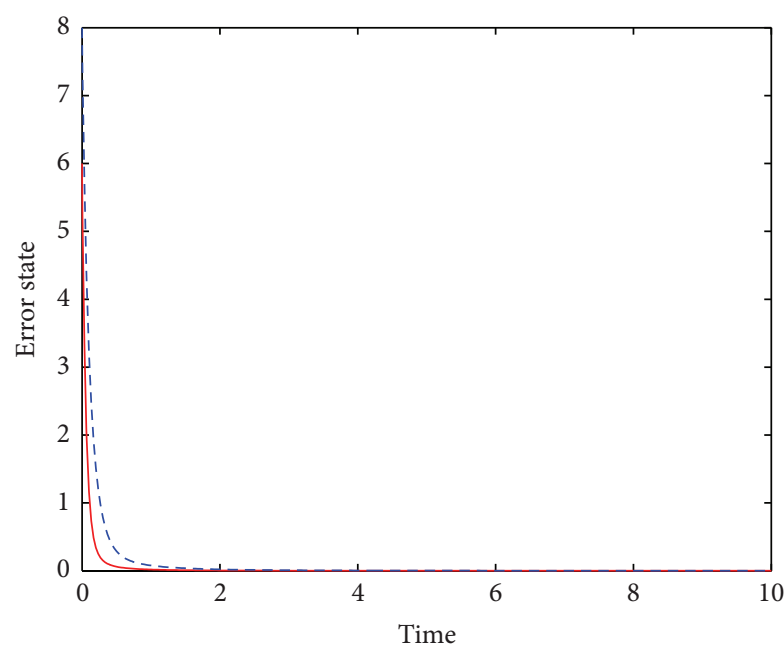

$X^{1}$, the 1st element of error state

-- $X^{2}$, the 2nd element of error state

FIGURE 3: The figure of error state.

\section{Conflict of Interests}

The authors declare that there is no conflict of interests regarding the publication of this paper.

\section{Acknowledgment}

This work was supported by the National Natural Science Foundation of China under Grant no. 61273158.

\section{References}

[1] D. Liberzon, Switching in Systems and Control, Birkhäuser, Boston, Mass, USA, 2003.

[2] R. Shorten, F. Wirth, and D. Leith, "A positive systems model of TCP-like congestion control: asymptotic results," IEEE/ACM Transactions on Networking, vol. 14, no. 3, pp. 616-629, 2006.

[3] A. Jadbabaie, J. Lin, and A. S. Morse, "Coordination of groups of mobile autonomous agents using nearest neighbor rules," IEEE Transactions Automatic Control, vol. 48, no. 6, pp. 988-1001, 2003.

[4] E. Hernandez-Vargas, P. Colaneri, R. Middleton, and F. Blanchini, "Discrete-time control for switched positive systems with application to mitigating viral escape," International Journal of Robust and Nonlinear Control, vol. 21, no. 10, pp. 1093-1111, 2011.

[5] L. Gurvits, R. Shorten, and O. Mason, "On the stability of switched positive linear systems," IEEE Transactions on Automatic Control, vol. 52, no. 6, pp. 1099-1103, 2007.

[6] X. Xue and Z. Li, "Asymptotic stability analysis of a kind of switched positive linear discrete systems," IEEE Transactions on Automatic Control, vol. 55, no. 9, pp. 2198-2203, 2010.

[7] O. Mason and R. Shorten, "On linear copositive Lyapunov functions and the stability of switched positive linear systems," IEEE Transactions on Automatic Control, vol. 52, no. 7, pp. 13461349, 2007.

[8] H. Alonso and P. Rocha, "A general stability test for switched positive systems based on a multidimensional system analysis,"
IEEE Transactions on Automatic Control, vol. 55, no. 11, pp. 2660-2664, 2010.

[9] L. Fainshil, M. Margaliot, and P. Chigansky, "On the stability of positive linear switched systems under arbitrary switching laws," IEEE Transactions on Automatic Control, vol. 54, no. 4, pp. 897-899, 2009.

[10] X. Liu and C. Dang, "Stability analysis of positive switched linear systems with delays," IEEE Transactions on Automatic Control, vol. 56, no. 7, pp. 1684-1690, 2011.

[11] A. Benzaouia, A. Hmamed, and F. Tadeo, "Stabilisation of controlled positive delayed continuous-time systems," International Journal of Systems Science, vol. 41, no. 12, pp. 1473-1479, 2010.

[12] A. Benzaouia and F. Tadeo, "Stabilization of positive switching linear discrete-time systems," International Journal of Innovative Computing, Information and Control, vol. 6, no. 6, pp. 24272437, 2010.

[13] C. Sun, X. Jing, H. Yan, and S. Xing, "State observer design for switched linear systems," Journal of Shenyang Jianzhu University (Natural Science), vol. 23, no. 1, pp. 166-169, 2007.

[14] A. Tanwani, H. Shim, and D. Liberzon, "Observability for switched linear systems: characterization and observer design," IEEE Transactions on Automatic Control, vol. 58, no. 4, pp. 891904, 2013.

[15] W. Xiang, J. Xiao, and M. N. Iqbal, "Robust observer design for nonlinear uncertain switched systems under asynchronous switching," Nonlinear Analysis: Hybrid Systems, vol. 6, no. 1, pp. 754-773, 2012.

[16] M. Xiang and Z. Xiang, "Observer design of switched positive systems with time-varying delays," Circuits, Systems, and Signal Processing, vol. 32, no. 5, pp. 2171-2184, 2013.

[17] M. Rami, U. Helmke, and F. Tadeo, "Positive observation problem for linear time-delay positive systems," in Proceedings of the 15th Mediterranean Conference on Control and Automation, pp. $1-6$, July 2007.

[18] M. Rami and F. Tadeo, "Positive observation problem for linear discrete positive systems," in Proceedings of the 45th IEEE Conference on Decision and Control (CDC '06), pp. 4729-4733, December 2006.

[19] Q. Huang, "Observer design for discrete-time positive systems with delays," in Proceedings of the International Conference on Intelligent Computation Technology and Automation (ICICTA '08), pp. 655-659, October 2008.

[20] P. Li, J. Lam, and Z. Shu, "Positive observers for positive interval linear discrete-time delay systems," in Proceedings of the 48th IEEE Conference on Decision and Control (CDC '09), pp. 61076112, December 2009.

[21] Z. Shu, J. Lam, H. Gao, B. Du, and L. Wu, "Positive observers and dynamic output-feedback controllers for interval positive linear systems," IEEE Transactions on Circuits and Systems, vol. 55, no. 10, pp. 3209-3222, 2008.

[22] Y. G. Sun, "Delay-independent stability of switched linear systems with unbounded time-varying delays," Abstract and Applied Analysis, vol. 2012, Article ID 560897, 11 pages, 2012.

[23] J. N. Lu and G. Y. Zhao, "Stability analysis based on LMI for switched systems with time delay," Journal of Southern Yangtze University (Natural Science), vol. 5, no. 2, pp. 171-173, 2006.

[24] L. Y. Zhao and Z. Q. Zhang, "Stability analysis of a class of switched systems with time delay," Control and Decision, vol. 26, no. 7, pp. 1113-1116, 2011.

[25] J. Lian, C. $\mathrm{Mu}$, and P. Shi, "Asynchronous $H_{\infty}$ filtering for switched stochastic systems with time-varying delay," Information Sciences, vol. 224, pp. 200-212, 2013. 
[26] G. S. Zhai, H. Lin, and P. J. Antsaklis, "Quadratic stabilizability of switched linear systems with polytopic uncertainties," International Journal of Control, vol. 76, no. 7, pp. 747-753, 2003.

[27] L. X. Zhang, P. Shi, E. K. Boukas, and C. Wang, "Robust $l_{2}-$ $l_{\infty}$ filtering for switched linear discrete time-delay systems with polytopic uncertainties," IET Control Theory \& Applications, vol. 1, no. 3, pp. 722-730, 2007.

[28] A. Benzaouia, M. Ouladsine, A. Naamane, and B. Ananou, "Fault detection for uncertain delayed switching discrete-time systems," International Journal of Innovative Computing Information and Control, vol. 8, no. 12, pp. 8049-8062, 2012. 


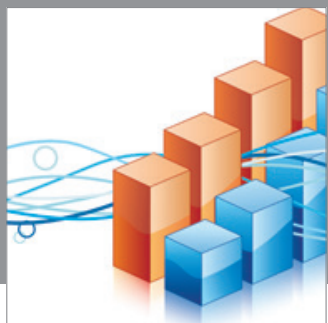

Advances in

Operations Research

mansans

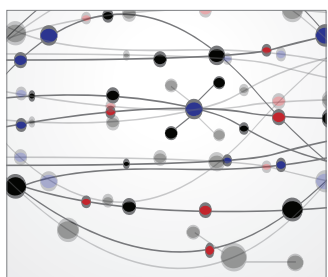

The Scientific World Journal
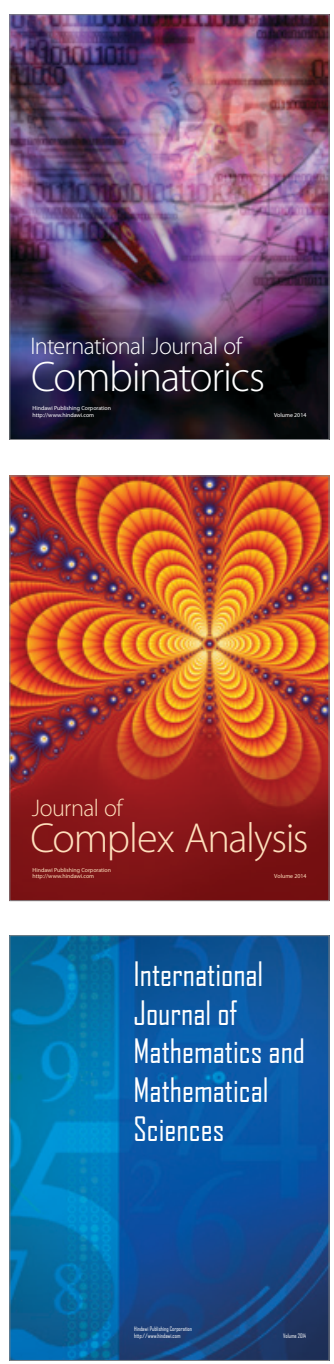
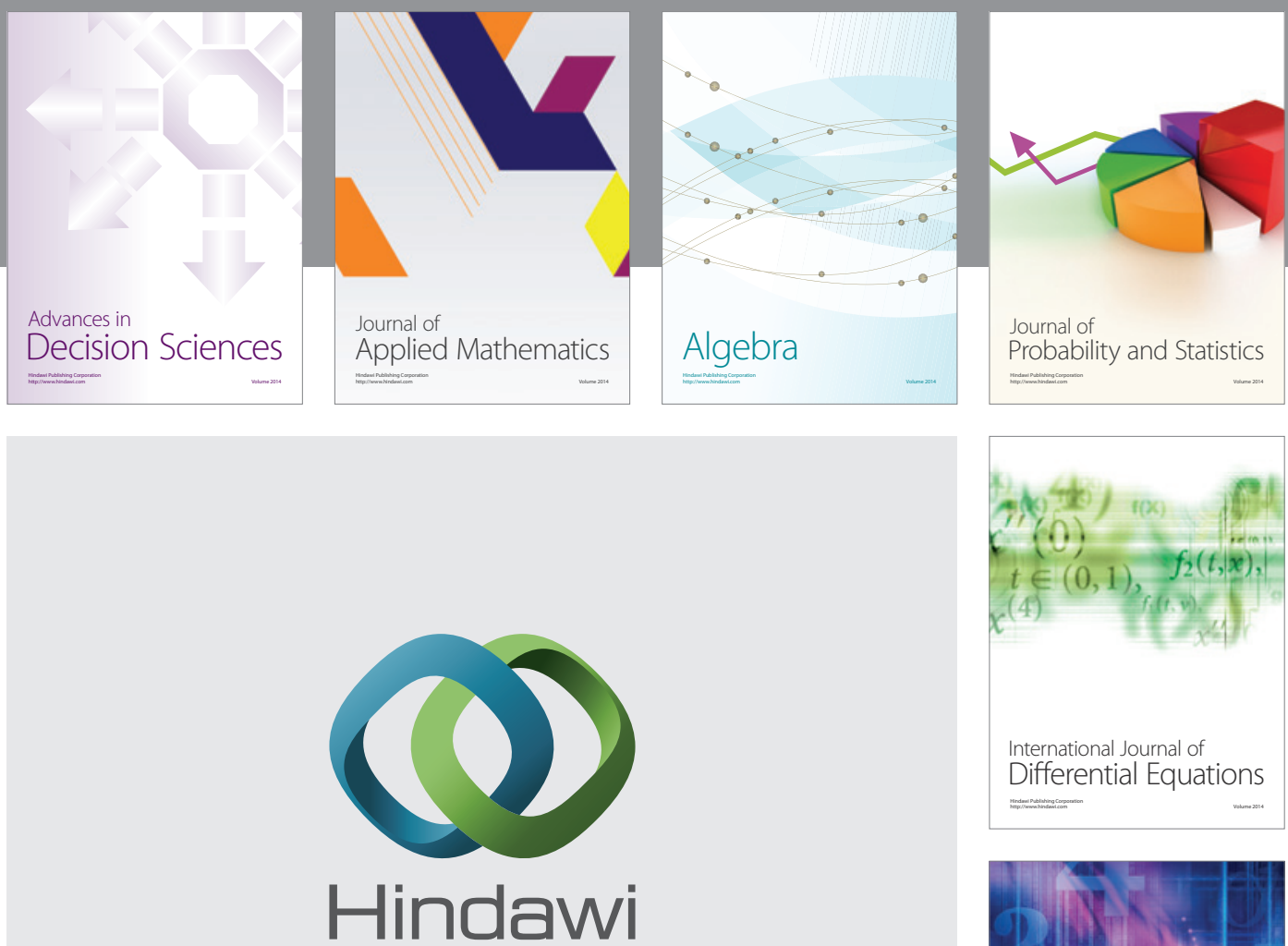

Submit your manuscripts at http://www.hindawi.com
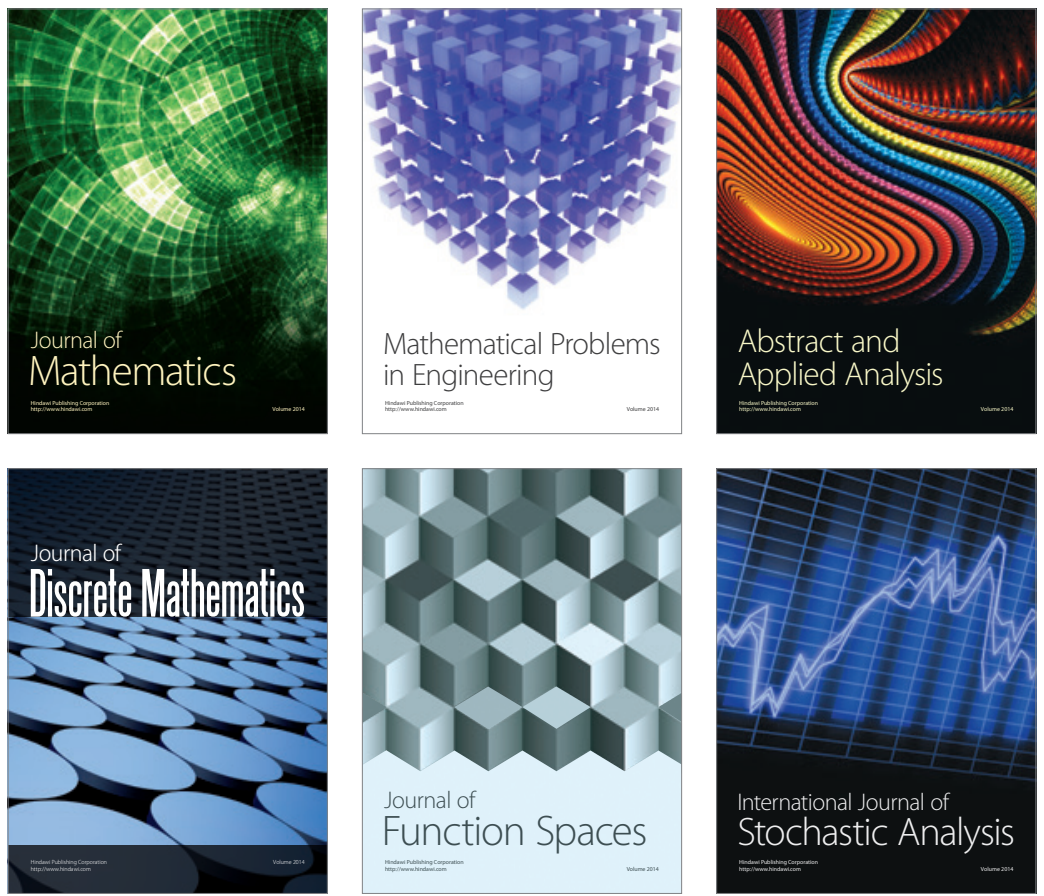

Journal of

Function Spaces

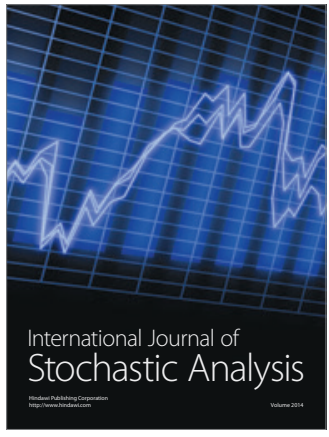

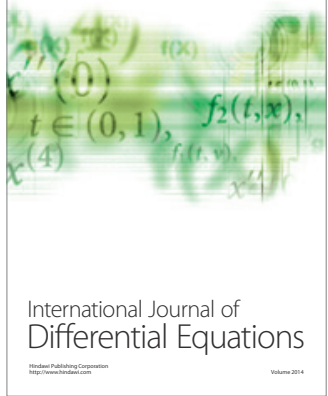
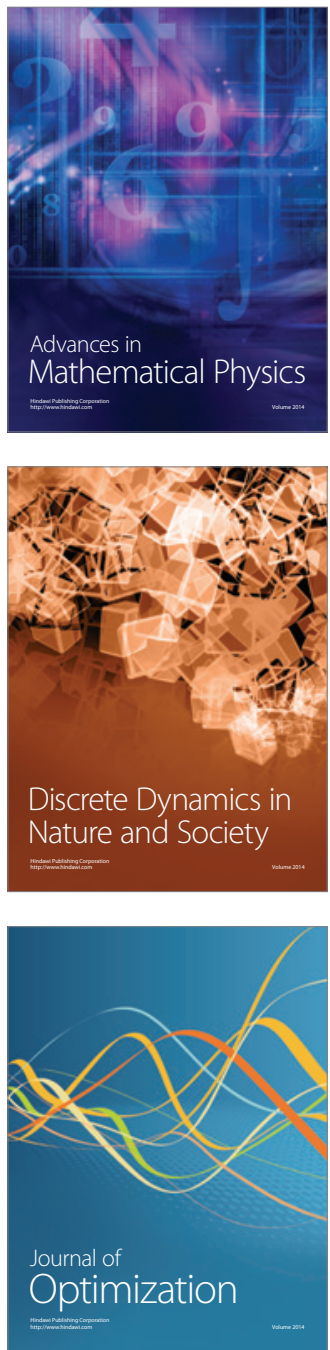